OPEN ACCESS

Edited by:

Zhe-Sheng Chen,

St. John's University, United States

Reviewed by:

Lihua Liu,

Fourth Hospital of Hebei Medical

University, China

Jingting Jiang,

First People's Hospital of

Changzhou, China

*Correspondence:

Yi Zhang

yizhang@zzu.edu.cn

Li Yang

fccyangl1@zzu.edu.cn

${ }^{\dagger}$ These authors have contributed equally to this work

Specialty section: This article was submitted to Molecular and Cellular Oncology,

a section of the journal

Frontiers in Oncology

Received: 12 November 2021

Accepted: 06 December 2021

Published: 06 January 2022

Citation:

Yu W, Liu F, Lei Q, Wu P, Yang L and Zhang $Y$ (2022) Identification of Key

Pathways and Genes Related to

Immunotherapy Resistance of LUAD

Based on WGCNA Analysis.

Front. Oncol. 11:814014.

doi: 10.3389/fonc.2021.814014

\section{Identification of Key Pathways and Genes Related to Immunotherapy Resistance of LUAD Based on WGCNA Analysis}

\author{
Weina Yu ${ }^{1,2,3+}$, Fengsen Liu ${ }^{1,2,3 \dagger}$, Qingyang Lei ${ }^{1,2,3}$, Peng Wu ${ }^{1,2,3}$, Li Yang ${ }^{1,2,3 *}$ \\ and Yi Zhang ${ }^{1,2,3,4 *}$
}

\begin{abstract}
${ }^{1}$ Biotherapy Center and Cancer Center, The First Affiliated Hospital of Zhengzhou University, Zhengzhou, China, ${ }^{2}$ Henan Key Laboratory for Tumor Immunology and Biotherapy, Zhengzhou University, Zhengzhou, China, ${ }^{3}$ State Key Laboratory of Esophageal Cancer Prevention and Treatment, Zhengzhou University, Zhengzhou, China, ${ }^{4}$ School of Life Sciences, Zhengzhou University, Zhengzhou, China
\end{abstract}

Immunotherapy resistance is a major barrier in the application of immune checkpoint inhibitors (ICl) in lung adenocarcinoma (LUAD) patients. Although recent studies have found several mechanisms and potential genes responsible for immunotherapy resistance, ways to solve this problem are still lacking. Tumor immune dysfunction and exclusion (TIDE) algorithm is a newly developed method to calculate potential regulators and indicators of $\mathrm{ICI}$ resistance. In this article, we combined TIDE and weighted gene coexpression network analysis (WGCNA) to screen potential modules and hub genes that are highly associated with immunotherapy resistance using the Cancer Genome Atlas (TCGA) dataset of LUAD patients. We identified 45 gene co-expression modules, and the pink module was most correlated with TIDE score and other immunosuppressive features. After considering the potential factors in immunotherapy resistance, we found that the pink module was also highly related to cancer stemness. Further analysis showed enriched immunosuppressive cells in the extracellular matrix (ECM), immunotherapy resistance indicators, and common cancer-related signaling pathways in the pink module. Seven hub genes in the pink module were shown to be significantly upregulated in tumor tissues compared with normal lung tissue, and were related to poor survival of LUAD patients. Among them, THY1 was the gene most associated with TIDE score, a gene highly related to suppressive immune states, and was shown to be strongly expressed in late-stage patients. Immunohistochemistry $(\mathrm{IHC})$ results demonstrated that THY1 level was higher in the progressive disease (PD) group of LUAD patients receiving a PD-1 monoclonal antibody $(\mathrm{mAb})$ and positively correlated with SOX9. Collectively, we identified that THY1 could be a critical biomarker in predicting $\mathrm{ICI}$ efficiency and a potential target for avoiding tumor immunotherapy resistance.

Keywords: LUAD, WGCNA, TIDE, immunotherapy resistance, cancer stem cell 


\section{INTRODUCTION}

Chemotherapy, radiotherapy, and surgery have dominated the cancer treatment field for years and did acquire considerable effects. However, these traditional therapies have noticeable limitations in treating patients with late-stage or metastatic malignant tumors and often cause severe side effects. To solve current problems, immunotherapies are emerging as promising methods by rebuilding immunosurveillance and stimulating tumor immune elimination (1). T cells are primary participants in tumor-associated immune response. Their infiltration, ratio, and functions influence antitumor immune response (2). Immune checkpoints [cytotoxic T lymphocyte-associated antigen-4 (CTLA-4), programmed cell death protein 1 (PD-1)] and immune checkpoint-related ligands [immune checkpoint-related ligands such as programmed cell death ligand 1 (PD-L1)] are critical parts in this process that promote or inhibit T-cell activity (3). As a result, ICIs are considered a promising strategy in reactivating antitumor immune responses by blocking immune checkpoints/immune checkpoint ligands. In recent decades, ICI therapy has been shown to be effective in patients with refractory tumors, especially for those late-stage patients who were unresponsive to traditional treatments (4). The United States Food and Drug Administration approved its application in solid tumors in 2011 (5). Anti-PD-1/PD-L1 therapy increased the overall survival to $40-50 \%$ at 5 years in advanced melanoma patients and is also becoming a promising method for non-smallcell lung cancer (NSCLC) patients $(6,7)$. The KEYNOTE-042 study indicated that in NSCLC patients whose PD-L1 tumor proportion score was $\geq 1 \%$, pembrolizumab monotherapy prolonged their survival for longer than with traditional chemotherapy (8). In addition, dual blockage of PD-1 and CTLA-4 using nivolumab and ipilimumab also showed better effects compared with chemotherapy (9).

However, ICI did not show the same effects in all malignant tumor patients, and its response rate in an unselected population as single agent is below $20 \%(7,10)$. More than one-half of patients are either unresponsive, or they relapse after a period of response, which seriously limits the effectiveness of ICI (11). ICI resistance can be generally divided into two sorts: primary resistance in which patients experience no significant effect in the initial period of ICI treatment; and secondary resistance (acquired drug resistance) in which initially, ICI can slow tumor progression, but patients later develop treatment failure (12). Two primary reasons for ICI resistance are the dysfunction of tumor infiltrating cytotoxic $\mathrm{T}$ cells and immunosuppressive factors, which hinder $\mathrm{T}$ cells from entering the tumor microenvironment (TME) $(13,14)$. Although many mechanisms were reported to be accountable for ICI resistance, there is still not an efficient method to predict it. Therefore, identification of predictive biomarkers for ICI response is crucial for determining appropriate patients for and predicting efficacy of ICI. TIDE is a computational method developed by Jiang et al. and is used for forming a tumor immune evasion model and calculating potential regulators and indicators in ICI resistance through integrating the expression features of $\mathrm{T}$ cell dysfunction and $\mathrm{T}$ cell exclusion, as well as pre-treatment tumor profiles (15). To identify potential biomarkers associated with immunotherapy, especially ICI resistance, WGCNA was also adopted in this study. WGCNA is a newly developed bioinformatics method to process medical data. Various researchers have utilized this method to analyze the gene expressions in different samples, depict correlations, and identify potential co-expressed genes or targets (16-18).

Self-renewal capacity and long-term persistence are critical properties of cancer stem cells (CSCs), and help them to maintain immune evasion, epithelial-mesenchymal transition (EMT), signaling pathway regulation, multidrug resistance, and pro-tumor immunity (19). CSCs express low levels of cancerassociated antigens, immune stimulatory molecules (CD86, CD40, and MHC II), and high levels of immune checkpoint proteins (20). Recent studies put forward that cancer cell stemness could be represented by clusters of core genes (2123). Based on these gene sets, Miranda et al. found pervasive negative associations between cancer cell stemness and anticancer immunity (24). They showed that stemness led to higher intratumoral heterogeneity and restrained antitumor immune response, which result in poor outcome for malignant tumor patients. Research showed that complex interactions between CSCs and immune cells in the TME-induced immunotherapy resistance by promoting anti-apoptotic pathways, inducing EMT, and enhancing immune tolerance (25-27).

After taking into consideration both TIDE score and stemness index, we identified a potential gene module (pink) which was highly related to $\mathrm{T}$ cell dysfunction and cancer cell stemness. Further analysis revealed strong enrichment of ECM remodeling, EMT, tumor invasion, tumor growth, and a positive relationship to immunosuppressive markers in the pink module. According to the gene significance of TIDE (GS.TIDE), we selected nine genes and observed their higher levels in tumor tissues and LUAD patients with poorer survival. Among them, THY1 (Thy-1 cell surface antigen) was selected as the key gene and was shown to be related to tumor stage, survival, and immune states of LUAD patients. Analysis of clinical samples showed a positive correlation between THY1 and classical cancer stemness marker SOX9. Moreover, the highest levels of THY1 were found in the PD group of PD-1 mAb-treated LUAD patients. These results provide the fundamental basis for further research of immunotherapy resistance and the discovery of new therapeutic target to refine ICI treatments of LUAD patients.

\section{MATERIALS AND METHODS}

\section{Data Processing}

We obtained gene expression data (FPKM format) and relevant clinical characteristics of 526 LUAD samples from the Cancer Genome Atlas (TCGA) database (https://www.cancer.gov/tcga). After excluding patients that lacked key clinical information, 513 patients were retained. TPM format conversion was performed for gene expression data in FPKM format for further analysis. We also obtained data for gene expression and survival of LUAD patients (GSE72094) from Gene Expression Omnibus (https:// 
www.ncbi.nlm.nih.gov/geo/). A total of 398 samples with complete clinical information were utilized in further analysis.

\section{Immunotherapy Response Prediction and Stemness Index}

TIDE score was shown to be more effective and accurate than current methods in predicting the immunotherapy response of melanoma and lung cancer patients. We downloaded LUAD patient-related TIDE scores from (http://tide.dfci.harvard.edu) (15). To bring cancer stemness into analysis, we used single-sample gene set enrichment analysis (ssGSEA) to process stemness-related gene sets (Benporath_ES1, Benporath_ES2, Bhattacharya_hESC, Shats_iPSC, Shats_Consensus, Benporath_Sox2, Kim_Myc, Smith_Human. Epithelial_ASC, Palmer_2012, Proliferative_Ben_Porath, Curated_genes) in malignant tumors obtained from previous articles (21-23, 28-32). The above data were included in the WGCNA analysis according to a previous report (33).

\section{Constructing Weighted Gene Co-Expression Networks and Identifying Modules Associated With Immunotherapy Resistance Score and Stemness Score of LUAD Patients}

WGCNA is used to group correlated genes pairwise into a model or network according to their similar expression profiles (34). In this article, WGCNA was adopted to identify the modules correlated with immunotherapy resistance and cancer stemness. Further filtration of 513 LUAD patients found that six patients lacked TIDE values or were outliers according to the original clustering tree at a height of 150,000. As a result, we ultimately included 507 samples in the WGCNA analysis. We chose the soft threshold $\beta=4$ (scale free $\mathrm{R}^{2}=0.88$ ) to construct a co-expression network. We then transformed the adjacency matrix into a topological overlap matrix to quantitatively describe the similarity. Next, we used cutreeDynamic function to perform the gene hierarchical clustering dendrograms and identify various co-expression modules. Correlations between modules and TIDE scores or other characteristics were assessed by Spearman test.

\section{Identification of Hub Genes}

GS and module membership (MM) were adopted to filter hub genes in the pink module. GS stands for the level of correlation between gene expression and designated features. MM stands for the correlation of the module eigengene and the gene expression profile. GS.TIDE represents the relevance of each module to the TIDE score. Among all the modules, the pink module had the highest GS.TIDE, which means the highest correlation with immune evasion. Therefore, we selected top 20 genes according to GS.TIDE for protein-protein interaction (PPI) network analysis and nine genes for further analysis. Among them, THY1 was the gene most associated with TIDE score in the pink module.

\section{Protein-Protein Interaction Analysis of Top 20 Hub Genes}

We performed a PPI analysis with top 20 hub genes using GeneMANIA website (https://genemania.org/search/homosapiens/) to depict their relationship in Co-expression, Physical Interactions, Co-localization, and Pathways.

\section{Gene Ontology and Kyoto Encyclopedia of Genes and Genomes Enrichment Analysis}

Clusterprofiler R package was adopted in Gene Ontology (GO) and Kyoto Encyclopedia of Genes and Genomes (KEGG) enrichment analysis using genes in the pink module. We used "adjusted $p<0.05$ " to identify significant GO terms and KEGG terms.

\section{Cell Infiltration Analysis}

xCell (http://xcell.ucsf.edu/) was used to obtain the relative expression level of 64 types of cells in the tumor microenvironment of LUAD patients using mRANseq data (TPM format) from TGCA in R software.

\section{Pathway Enrichment of the Pink Module}

Four gene set lists were downloaded from the nanoString website (https://www.nanostring.com/): the nCounter PanCancer Pathways panel, the nCounter ${ }^{\circledR}$ PanCancer Immune Profiling panel, the nCounter PanCancer Progression panel, and the nCounter ${ }^{\circledR}$ PanCancer IO $360^{\mathrm{TM}}$ Panel. These panels include detailed listings of genes, corresponding pathways, and immune types. ssGSEA and heatmap packages were used to analyze these four panels and displayed the results by LUAD patients.

\section{Kaplan-Meier Survival Analysis}

Gene expression data and corresponding clinical information from GSE72094 dataset were used to analyze the survival rate of each gene. We utilized Kaplan-Meier curve and log-rank test to estimate the prognostic significance of nine hub genes. We divided each gene into two groups according to the best cut point method using "surv_cutpoint" algorithm of the survminer R package.

\section{Immune Subtypes and Clinical Characteristics Analysis}

According to the research of Thorsson et al., the intratumor immune states could be divided into six subtypes: $\mathrm{C} 1$ (wound healing), C2 (IFN- $\gamma$ dominant), C3 (inflammatory), C4 (lymphocyte depleted), C5 (immunologically quiet), and C6 (TGF- $\beta$ dominant) (35). C1 has higher expression of angiogenic genes and Th2-type adaptive immune infiltration. C2 shows high M1/M2 macrophage polarization and activated function of antitumor $\mathrm{T}$ cells. C3 is related to Th17 and Th1 cells and could suppress tumor cell proliferation. C4 displays a prominent M2 macrophage signature and suppressed Th1. C5 exhibits the lowest lymphocyte and highest $\mathrm{M} 2$ responses. The highest TGF- $\beta$ signature is the feature of C6. We evaluated the expression level of THY1 on these six subtypes. We also assessed THY1 expression in different clinical stages of LUAD patients using gene expression data and clinical information from the TCGA dataset. Wilcoxon test was adopted to compare differences between groups.

\section{Clinical Samples}

Human LUAD tumor tissues were acquired from the First Affiliated Hospital of Zhengzhou University in 2020. All patients were pathologically diagnosed with LUAD through surgery or percutaneous pulmonary biopsy and failed chemotherapy or targeted therapy. All patients had a lung computerized tomography (CT) scan before receiving at least three cycles of 
PD-1 mAb treatment (baseline). Lung CT scan was also used to evaluate the therapeutic effects after using PD-1 mAb (first/second). The clinical information of the patients is shown in Table $\mathbf{1}$.

\section{Immunohistochemistry}

All patients agreed to informed consent before specimen collection. Tissues were fixed with $4 \%$ paraformaldehyde and embedded in paraffin. IHC was performed according to previous research (36). Tumor tissue slides were incubated with antihuman THY1 (ABCAM, Rabbit mAb, CAT: ab133350, 1:50) and anti-human SOX9 (ABCAM, Rabbit mAb, CAT: ab185966, $1: 1,000)$ at $4^{\circ} \mathrm{C}$ for overnight. Then, the slides were incubated with horseradish peroxidase-conjugated anti-rabbit/mouse antibody (ZSGB-BIO, SP-9000) for $30 \mathrm{~min}$ at room temperature. Subsequently, diaminobenzidine (ZSGB-BIO, ZLI-9018) and hematoxylin were used to visualize the protein and cell nucleus. Vectra Polaris Multispectral Imaging and Whole Slide Scanning System (PerkinElmer, Vectra) was used to screen the slides. IHC staining score of each protein was evaluated by imageJ software using IHC toolbox.

\section{Statistical Analysis}

Wilcoxon test is used to compare the differences between groups of classified variable. Spearman analysis is used to calculate the correlation between continuous variables. The above collected data was analyzed using $\mathrm{R}$ software version 3.6.1 (https://www.rproject.org). Differences between groups were analyzed using $\mathrm{t}$ test. Pearson's correlation analysis was used to calculate correlation coefficients ( $\mathrm{r}$ ) using Graphpad Prism 7. With all statistical methods, $p<0.05$ was considered statistically significant.

\section{RESULT}

\section{WCGNA Analysis and Modules Significance Calculation}

The gene expression matrix of 7,298 preprocessed genes derived from LUAD patients in the TCGA dataset was used for WGCNA with $\mathrm{R}$ package. After excluding patients that lacked critical characteristics, 507 samples were utilized for further analysis.

TABLE 1 | The summary clinical information of the samples.

\begin{tabular}{lc}
\hline Characteristics & LUAD Patients $(\mathbf{n}=\mathbf{6})$ \\
\hline Age, year (mean $\pm S D)$ & $63.50 \pm 8.12$ \\
Male, $n(\%)$ & $2(33.3)$ \\
Female, $n(\%)$ & $4(66.7)$ \\
TNM stage, $n$ (\%) & \\
I & $0(0)$ \\
II & $1(16.7)$ \\
III & $3(50)$ \\
IV & $2(33.3)$ \\
Outcome, $n$ & \\
PD & 2 \\
SD & 2 \\
PR & 2
\end{tabular}

$S D$, standard deviation; $L U A D$, lung adenocarcinoma; $P D$, progressive disease; $S D$, stable disease; PR, partial response.
Sample dendrogram and trait heatmap are displayed in Figure 1A. To ensure high-scale independence (near 0.9), we set $\beta=4$ in this analysis (Figure 1B). After obtaining the $\beta$ value, we constructed an adjacency matrix and topological overlap matrix (Figures 1C, D). On the basis of average hierarchical clustering and dynamic tree clipping, we obtained 45 modules in total (Figure 2A). Then, we calculated the correlation coefficients between each module and the sample characteristics related to TIDE, cancer stemness markers, and various immune-related characteristics (Figures 2B, C). According to GS.TIDE, we found that the pink module had the highest correlation with TIDE (cor $=0.56 ; p=2 \mathrm{e}-43$ ). In addition, the pink module showed a strong correlation with various cancer stemness markers and immunosuppressive features, such as Exclusion (cor $=0.55 ; p=$ $4 \mathrm{e}-42$ ) and cancer-associated fibroblast (CAF) (cor $=0.9 ; p=3 \mathrm{e}-$ 189) (Figure 2B). As a result, this module was selected as a critical module for the possible relationship between cancer stemness and immunotherapy resistance. GS.TIDE was used to filter the top 20 hub genes in the pink module (Table 2). We adopted the PPI network with top 20 hub genes using GeneMANIA and found a strong relationship in expression, physical interaction, and pathways between hub genes (Figure 2D).

\section{Functional Annotation of Genes in the Pink Module}

The pink module contains 274 genes. The results of the GO enrichment analysis and KEGG enrichment analysis of these 274 genes are shown in Figures 3A, B. Results showed that genes in the pink module were strongly enriched in the ECM organization. Enriched items in the cellular component were also related to the ingredients of ECM and focal adhesion (Figure 3A). Results from KEGG analysis showed a similar conclusion (Figure 3B). Top 15 GO items of the hub genes in the pink module are shown in Table 3.

To further investigate the characteristics of these genes, we utilized gene set lists downloaded from nanoString and identified higher enrichment of genes in the immunosuppressive items and tumor-associated pathways and functions (Figures 3C-F). For example, the pink module was associated with senescence, adhesion, and transporter functions and was also enriched with tumor intrinsic factors and stromal factors. A cluster of cancer-related pathways were involved, such as JAK-STAT, Wnt, PI3K, MAPK, and RAS. More importantly, the enrichment of metastasis, cancer metabolism, ECM remodeling, EMT, tumor invasion, tumor growth, angiogenesis, and ECM layers was heightened in the pink module. Highly positive correlations were found between the pink module and cancer stemness markers, such as Benporath-Nanog (Cor $=0.468, p=0 \mathrm{e}+00)$, Palmer_2012 (Cor $=0.331, p=2.452 \mathrm{e}-14)$, and Curated_genes (Cor $=0.326, p=7.074 \mathrm{e}-14)$ (Figure 4A).

It has been reported that the occurrence of immunotherapy resistance is associated with the upregulation of suppressive characteristics in TME and downregulation of immune stimulators (37). To support the hypothesis that the pink module is associated with immune evasion, we performed a correlation analysis between the pink module and immunosuppressive markers 
A

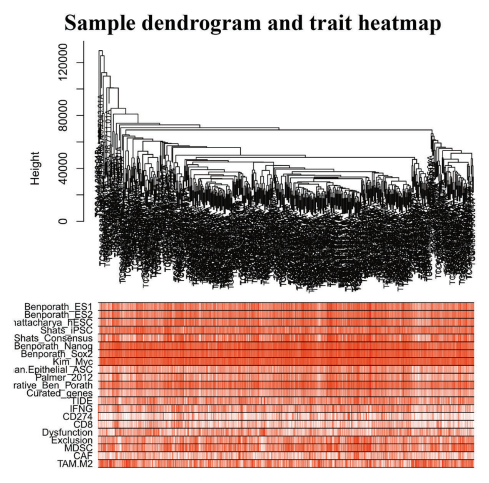

C

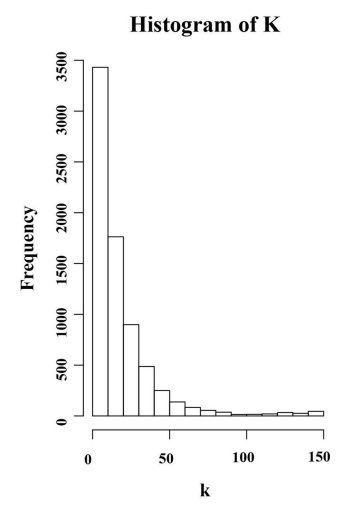

B

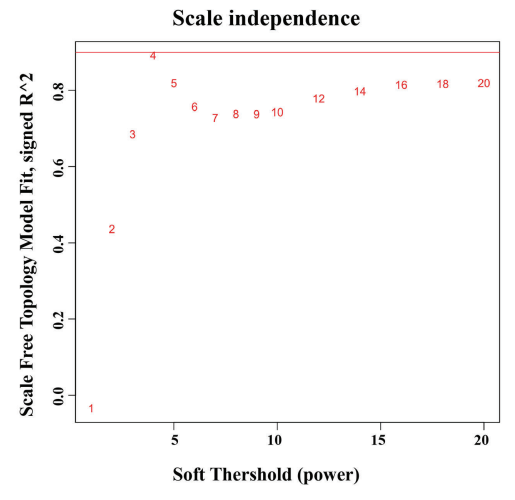

D

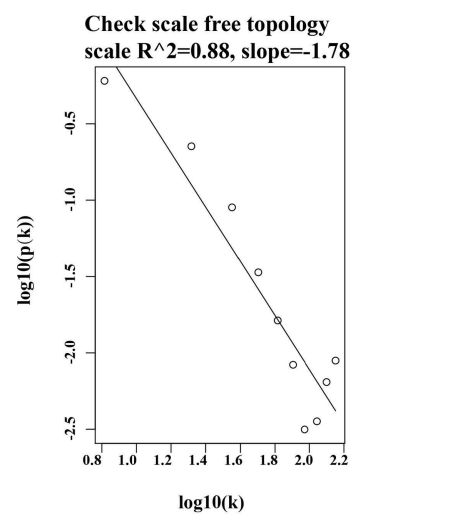

FIGURE 1 | Sample dendrogram and soft-thresholding values estimation. (A) Sample dendrogram and trait heatmap of 507 samples. The upper 12 traits are representative marker of cancer stemness, and the lower 9 traits are common evaluation index of immunotherapy. (B) Analysis of the scale-free index for various soft-threshold powers ( $\beta$ ). (C, D) The scale free topology when $\beta=4$.

or immunostimulatory makers. We found that pink module genes are positively correlated with immunosuppressive markers and cells, such as Tr1, Th2, iTreg, CAF, and CD274 (Figure 4B). Correspondingly, immune-stimulating cells and markers, like Th17, effector-memory and Gamma-delta $\mathrm{T}$ cells, and neutrophils, were negatively correlated with the pink module (Figure 4C). These results demonstrated that the pink module is a set of genes that potently correlated with cancer stemness and immunosuppressive characteristics.

\section{Identification and Validation of Hub Genes}

In WCGNA Analysis and Modules Significance Calculation we filtered the top 20 hub genes in the pink module according to GS.TIDE. To shrink the range, we ranked genes on the base of GS.TIDE and selected nine genes from them (THY1, COL5A1, COL1A2, COL3A1, COL1A1, COL5A2, MMP2, COL6A3, EMILIN1). The expression levels and clinical information of these nine genes were obtained from the TCGA and GEO database. Higher expression levels were found in the tumor tissues than in the corresponding normal tissues, except for EMILIN1 and MMP2 (Figure 5A). We then used a KaplanMeier plotter to analyze patient survival and found that $T H Y 1$, COL5A1, COL1A2, COL3A1, COL1A1, COL5A2, COL6A3 exhibited excellent diagnostic efficiency in LUAD patients
(Figure 5B). These results indicated the strong clinical significance of THY1, COL5A1, COL1A2, COL3A1, COL1A1, COL5A2, COL6A3.

\section{Clinical Characteristics of THY1}

To find a more targeted gene, we filtered these hub genes according to GS.TIDE, expression levels in cancer, and survival probability, and selected THY1 as the key gene in the pink module. We then analyzed THY1 based on the theory of Thorsson et al. and observed that THY1 was highly expressed in the wound-healing subtype (C1) and TGF- $\beta$ dominant subtype (C6) (Figure 6A), which means that THY1 is an immunosuppressive participant in malignant tumors. Moreover, clinical characteristics analysis showed that tumor stages were highly associated with THY1 expressions, which were highest in LUAD patients in stage iii (Figure 6B). Correlation analysis revealed that THY1 had a strong positive correlation with cancer stemness markers, as well as immunosuppressive markers and cells, especially with the TIDE score (Cor $=0.578, p=4.293 \mathrm{e}-50$ ) (Figures 6C, D). The negative correlation between THY1 and immune stimulators were also apparent (Figure 6E). These results implied that THY1 mediates immunotherapy resistance by enhancing immunosuppressive function as well as limiting the antitumor effects of immune stimulators. 


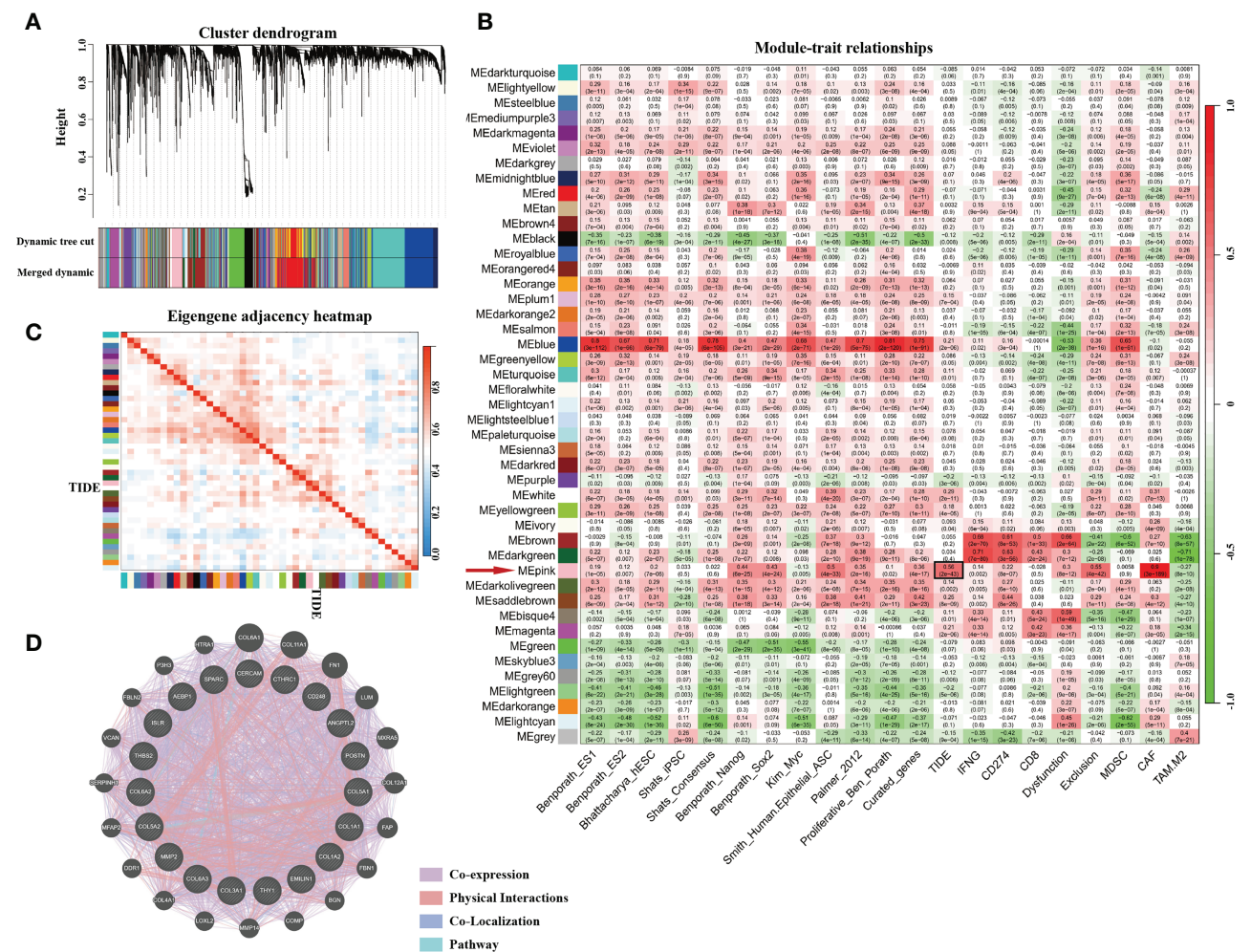

FIGURE 2 | The genes enrichment and module identification. (A) Hierarchical clustering dendrogram of co-expressed genes in LUAD patients. A total of 45 modules were identified, and each colored row stands for a module with a cluster of highly connected genes. (B) Correlation coefficient between consensus module eigengenes and TIDE score, cancer stemness, or other immune-related characteristics. (C) Heatmap plot of the adjacent modules. (D) Protein-protein interactions of top 20 hub genes. The strength of the relationship is associated with the thickness of the colorful lines.

TABLE 2 | The top 20 hub genes in pink module.

\begin{tabular}{llccr}
\hline Gene symbol & Module color & GS.TIDE & p.GS.TIDE & MM.PINK \\
\hline THY1 & pink & 0.565112 & $4.07 \mathrm{E}-44$ & 0.863729 \\
COL5A1 & pink & 0.561903 & $1.56 \mathrm{E}-43$ & 0.909754 \\
COL1A2 & pink & 0.545879 & $1.03 \mathrm{E}-40$ & 0.900204 \\
COL3A1 & pink & 0.53608 & $4.63 \mathrm{E}-39$ & 0.871879 \\
COL1A1 & pink & 0.536067 & $4.08 \mathrm{E}-195$ \\
COL5A2 & pink & 0.535168 & $1.86 \mathrm{E}-184$ \\
MMP2 & pink & 0.528323 & $1.08 \mathrm{E}-158$ \\
COL6A3 & pink & 0.52624 & $6.55 \mathrm{E}-39$ & $2.33 \mathrm{E}-148$ \\
EMILIN1 & pink & 0.520856 & $8.60 \mathrm{E}-38$ & $1.14 \mathrm{E}-178$ \\
CTHRC1 & pink & 0.519758 & $1.86 \mathrm{E}-37$ & $1.40 \mathrm{E}-157$ \\
CERCAM & pink & 0.519243 & $1.34 \mathrm{E}-36$ & 0.870475 \\
SPARC & pink & 0.517456 & $1.99 \mathrm{E}-36$ & 0.880475 \\
POSTN & pink & $2.40 \mathrm{E}-36$ & 0.828496 \\
ANGPTL2 & pink & 0.516439 & $4.56 \mathrm{E}-36$ & 0.714135 \\
COL6A2 & pink & 0.51307 & 0.686384 \\
AEBP1 & pink & 0.511227 & $6.56 \mathrm{E}-36$ & 0.898107 \\
THBS2 & pink & 0.508668 & $2.17 \mathrm{E}-35$ & 0.803405 \\
ISLR & pink & 0.506547 & $7.16 \mathrm{E}-35$ & $2.24 \mathrm{E}-72$ \\
CD248 & pink & 0.504474 & $1.02 \mathrm{E}-34$ & 0.835944 \\
BGN & pink & 0.499077 & $2.13 \mathrm{E}-34$ & 0.794625 \\
\end{tabular}

GS, gene significance; MM, module membership. 


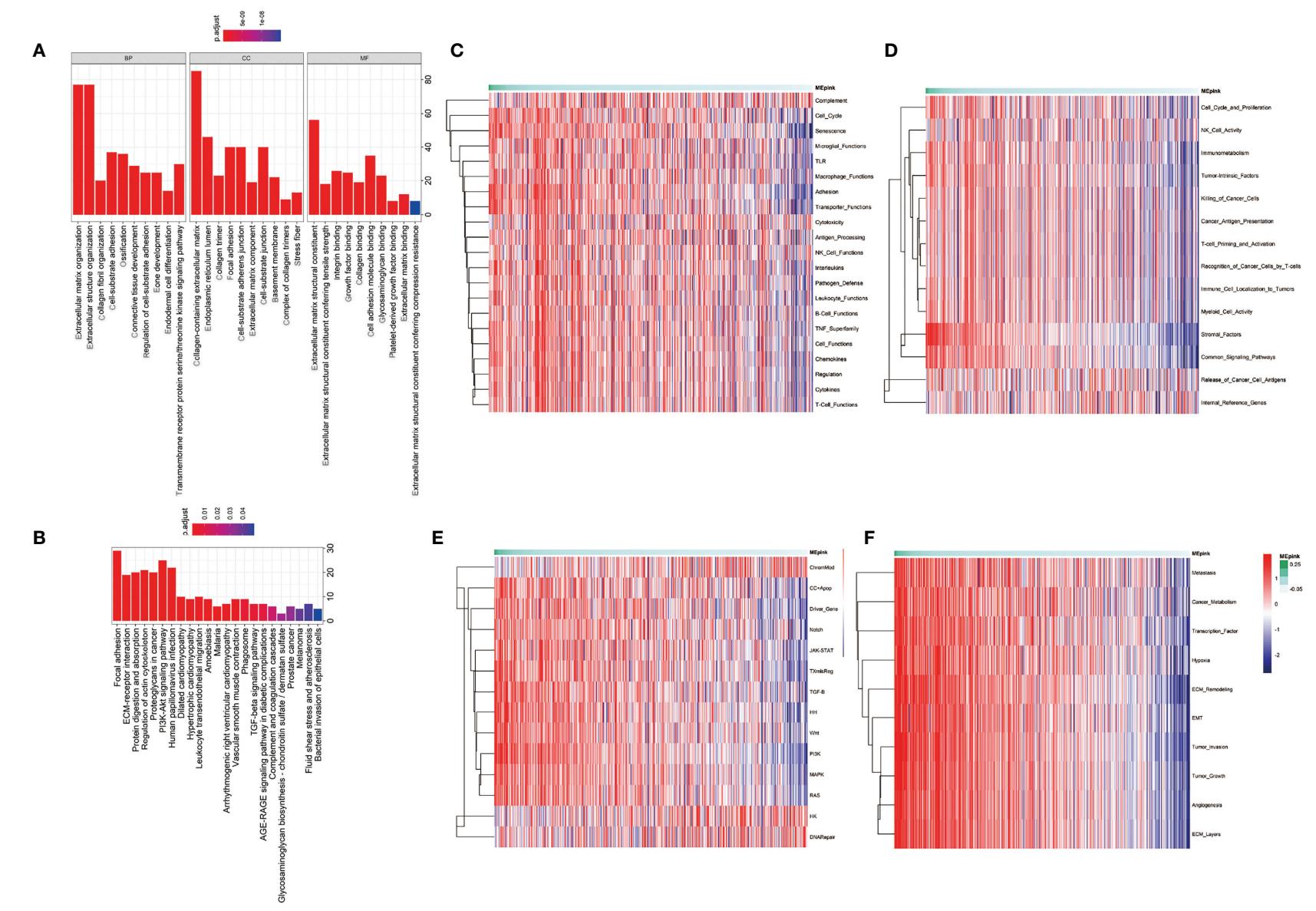

FIGURE 3 | Enrichment analysis of the significant genes in the pink module. (A) Gene ontology enrichment including biological process (BP), cellular component (CC), and molecular function (MF). (B) KEGG enrichment of significant genes in pink module. (C-F) Pathway enrichment of significant genes in pink module. (C) is the pathways that participate in immune profiling. (D) is the pathways in IO.unity. (E) is the common pathways in human physiological process. (F) is the pathways of cancer progression.

\section{Clinical Validation of THY1 and SOX9 in LUAD Patients}

In order to validate the hypothesis that THY1 is related to cancer stemness and immunotherapy resistance, we performed IHC staining on six tissue samples of LUAD patients who received at least three cycles of PD-1 mAb treatments using THY1 and SOX9 antibodies. The clinicopathological data are presented in Table 1. Lung CT examination was used to evaluate the efficacy of PD-1 mAb therapy, and the result prior to the first dose was used as the baseline. The results of the CT scan showed that two patients displayed $\mathrm{PD}$, two patients displayed stable disease (SD), and the remaining two patients acquired partial response (PR) (Figure 7C). IHC results proved that THY1 expression was highest in the $\mathrm{PD}$ group and lowest in the $\mathrm{PR}$ group. Moreover, THY1 expression was positively related to the level of SOX9, a classical biomarker of cancer stemness (38) (Figures 7A, B). However, the IHC scores of THY1 and SOX9 between PR group and SD group showed no statistical difference. These results indicated a strong relationship between THY1 and the therapeutic resistance of $\mathrm{PD}-1 \mathrm{mAb}$, which was consistent with the above analysis. Furthermore, THY1 levels also positively correlated with cancer stemness. This means that THY1 could be used as a possible biomarker in predicting the efficacy of PD$1 \mathrm{mAb}$.

\section{DISCUSSION}

The fact that ICI treatments are ineffective for some malignant tumor patients prompts research for the underlying molecular mechanisms and ways to refine it. As a newly developed computational method, TIDE has been used to model tumor immune evasion by evaluating the interactions between candidate genes and the characteristics of T cells in LUAD and melanoma (15). A higher TIDE score is related to a higher likelihood of immune escape by tumors and lower response rate to ICI treatment. As a result, TIDE score is now becoming a more reasonable and accurate method in assessing the outcome of 
TABLE 3 | The top 15 GO items of genes in the pink module.

\begin{tabular}{|c|c|c|c|}
\hline $\begin{array}{l}\text { GO- } \\
\text { ID }\end{array}$ & $\begin{array}{c}p- \\
\text { value }\end{array}$ & Description & Genes in the test list \\
\hline 5578 & $\begin{array}{l}3.30 \mathrm{E}- \\
20\end{array}$ & $\begin{array}{l}\text { Proteinaceous } \\
\text { extracellular matrix }\end{array}$ & $\begin{array}{l}\text { POSTN|SPARC|LUM|MMP2|BGN|COL1A1|ADAMTS2|COL3A1|VCAN|COL1A2|COL5A1|COL5A2|TIMP2|COL6A3| } \\
\text { ANGPTL2|EMILIN1|FBN1 }\end{array}$ \\
\hline 31012 & $\begin{array}{l}7.25 \mathrm{E}- \\
20\end{array}$ & Extracellular matrix & $\begin{array}{l}\text { POSTN|SPARC|LUM|MMP2|BGN|COL1A1|ADAMTS2|COL3A1|VCAN|COL1A2|COL5A1|COL5A2|TIMP2|COL6A3| } \\
\text { ANGPTL2|EMILIN1|FBN1 }\end{array}$ \\
\hline 44421 & $\begin{array}{l}3.67 \mathrm{E}- \\
15\end{array}$ & Extracellular region part & $\begin{array}{l}\text { POSTN|SPARC|LUM|MMP2|BGN|AEBP1|INHBA|COL1A1|ADAMTS2|COL3A1|VCAN|COL1A2|COL5A1|COL5A2|TIMP2| } \\
\text { COL6A3|ANGPTL2|EMILIN1|FBN1 }\end{array}$ \\
\hline 44420 & $\begin{array}{l}2.27 \mathrm{E}- \\
13\end{array}$ & Extracellular matrix part & COL1A1|COL3A1|SPARC|COL1A2|COL5A1|LUM|COL5A2|TIMP2|COL6A3|FBN1 \\
\hline 5583 & $\begin{array}{l}3.82 \mathrm{E}- \\
13\end{array}$ & Fibrillar collagen & COL1A1|COL3A1|COL1A2|COL5A1|LUM|COL5A2 \\
\hline 5201 & $\begin{array}{l}6.99 E- \\
13\end{array}$ & $\begin{array}{l}\text { Extracellular matrix } \\
\text { structural constituent }\end{array}$ & COL1A1|COL3A1|COL1A2|COL5A1|LUM|COL5A2|BGN|EMILIN1|FBN1 \\
\hline 30199 & $\begin{array}{l}1.47 \mathrm{E}- \\
12\end{array}$ & $\begin{array}{l}\text { Collagen fibril } \\
\text { organization }\end{array}$ & COL1A1|ADAMTS2|COL3A1|COL1A2|COL5A1|LUM|COL5A2 \\
\hline 48856 & $\begin{array}{l}1.86 \mathrm{E}- \\
12\end{array}$ & $\begin{array}{l}\text { Anatomical structure } \\
\text { development }\end{array}$ & $\begin{array}{l}\text { PDGFRB|TAGLN|POSTN|SPARC|UNC5B|MMP2|BGN|AEBP1|THY1|INHBA|ANTXR1|COL1A1|ACTA2|ADAMTS2|COL3A1| } \\
\text { VCAN|COL1A2|COL5A1|COL5A2|ITGA11|TIMP2|COL6A3|FBN1 }\end{array}$ \\
\hline 5576 & $\begin{array}{l}2.27 \mathrm{E}- \\
12\end{array}$ & Extracellular region & $\begin{array}{l}\text { POSTN|SPARC|LUM|MMP2|BGN|AEBP1|INHBA|THBS2|COL1A1|ADAMTS2|COL3A1|VCAN|COL1A2|COL5A1|COL5A2| } \\
\text { TIMP2|COL6A3|ANGPTL2|EMILIN1|MXRA5|FBN1 }\end{array}$ \\
\hline 30198 & $\begin{array}{l}3.94 \mathrm{E}- \\
12\end{array}$ & $\begin{array}{l}\text { Extracellular matrix } \\
\text { organization }\end{array}$ & COL1A1|ADAMTS2|POSTN|COL3A1|COL1A2|COL5A1|LUM|COL5A2|EMILIN1 \\
\hline 32502 & $\begin{array}{l}5.15 \mathrm{E}- \\
12\end{array}$ & Developmental process & $\begin{array}{l}\text { PDGFRB|TAGLN|POSTN|SPARC|UNC5B|MMP2|BGN|AEBP1|THY1|INHBA|ANTXR1|COL1A1|ACTA2|ADAMTS2|COL3A1| } \\
\text { VCAN|COL1A2|COL5A1|COL5A2|ITGA11|TIMP2|COL6A3|ANGPTL2|FBN1 }\end{array}$ \\
\hline 5581 & $\begin{array}{l}5.15 \mathrm{E}- \\
12\end{array}$ & Collagen & COL1A1|COL3A1|COL1A2|COL5A1|LUM|COL5A2|COL6A3 \\
\hline 7275 & $\begin{array}{l}1.35 \mathrm{E}- \\
11\end{array}$ & $\begin{array}{l}\text { Multicellular organismal } \\
\text { development }\end{array}$ & $\begin{array}{l}\text { PDGFRB|TAGLN|POSTN|SPARC|UNC5B|MMP2|BGN|AEBP1|THY1|INHBA|COL1A1|ACTA2|ADAMTS2|COL3A1|VCAN| } \\
\text { COL1A2|COL5A1|COL5A2|ITGA11|TIMP2|COL6A3|ANGPTL2|FBN1 }\end{array}$ \\
\hline 48731 & $\begin{array}{l}5.21 \mathrm{E}- \\
11\end{array}$ & System development & $\begin{array}{l}\text { PDGFRB|TAGLN|POSTN|SPARC|UNC5B|MMP2|BGN|AEBP1|THY1|INHBA|COL1A1|ADAMTS2|COL3A1|VCAN|COL1A2| } \\
\text { COL5A1|COL5A2|ITGA11|TIMP2|COL6A3|FBN1 }\end{array}$ \\
\hline 43062 & $\begin{array}{l}1.49 \mathrm{E}- \\
10\end{array}$ & $\begin{array}{l}\text { Extracellular structure } \\
\text { organization }\end{array}$ & COL1A1|ADAMTS2|POSTN|COL3A1|COL1A2|COL5A1|LUM|COL5A2|EMILIN1 \\
\hline
\end{tabular}

immunotherapy and prognosis of patients compared with the single evaluation of PD-L1 levels, tumor mutational burden, or IFN- $\gamma$ signature (39). To figure out which cluster of genes is potentially responsible for ICI resistance in LUAD patients, WGCNA was adopted to build a co-expression module and illuminate the complicated correlation between candidate genes and phenotypes. Highly co-expressed genes formed a module that can be used to assess the depth of the association with selected characteristics. Based on these concepts, we used the TIDE score and WGCNA to identify genes associated with immunotherapy resistance in LUAD patients, and picked the pink modules (274 genes), which was highly associated with the TIDE score. In addition, we introduced a cluster of cancer stemness markers (ES1/2, hESC, iPSC, Nanog, Sox2, Myc, et. al) into WGCNA analysis, and observed that these markers were positively correlated with the pink module (24) (Figure 2B). These results were in line with previous reports that CSCs mediate resistance to antitumor therapy, such as chemotherapy and radiotherapy, as well as immunotherapy (40-42).

In the exploration of immunotherapy resistance mechanism, cancer stemness has been a focus for years. Maccalli et al. proposed that one main reason for ICI treatment failure might be the immune-resistant ability of CSCs. Although few in number, CSCs remain in the tumor after treatment, reforming the tumor mass and promoting tumor progression (43). In the research by Miranda et al., they found that $\mathrm{CD} 8^{+} \mathrm{T}$ cells, natural killer (NK) cells, and B cells showed a strongly negative correlation with stemness for most cancer (24). These results proposed an association between stemness and suppressed antitumor immune response, which indicates worse clinical outcomes with malignant tumors. Complicated interactions between CSCs and other stromal cells, such as immune cells and fibroblasts, can lead to malignant biological properties such as EMT and result in immune evasion (44). In our analysis, top $15 \mathrm{GO}$ enrichment items of these genes showed a concentration on the organization and regulation of ECM, including EMT (Figures 3A, B).

The relationship between ECM remodeling and cancer stemness has already been reported. Elevated collagen-induced stiff ECM triggers cancer cell stem-like programming and metastatic dissemination in vivo. Suppression of collagen deposition could increase expression of lung differentiation markers (45). Liang et al. proposed that $\mathrm{CD} 44$, a transmembrane receptor for hyaluronic acid and many other ECM components, was a critical marker and regulator of cancer stemness (46). Furthermore, macrophagederived glycoprotein non-metastatic B facilitates the expansion of CSCs through CD44/IL-33 axis and promote metastasis in mouse tumor models (17). Moreover, ECM could facilitate immune evasion in CSCs by activating PI3K/AKT and recruiting immunosuppressive cells like tumor associated macrophages and Tregs (47-49). The relationship between tumor ECM, drug resistance, and immune suppression has been reported (50). 
A
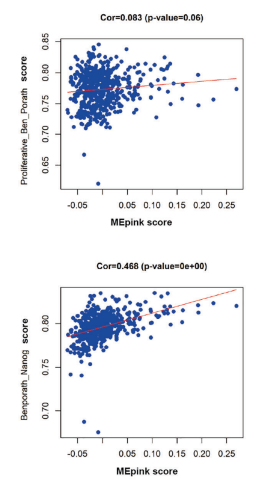

B

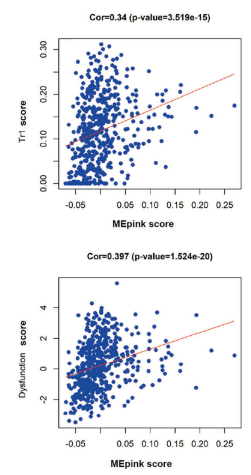

C
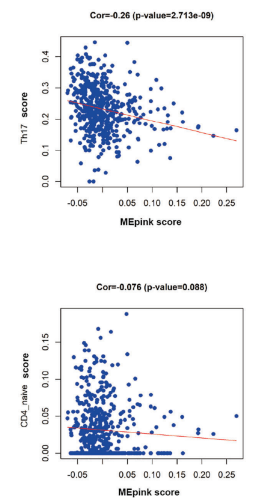
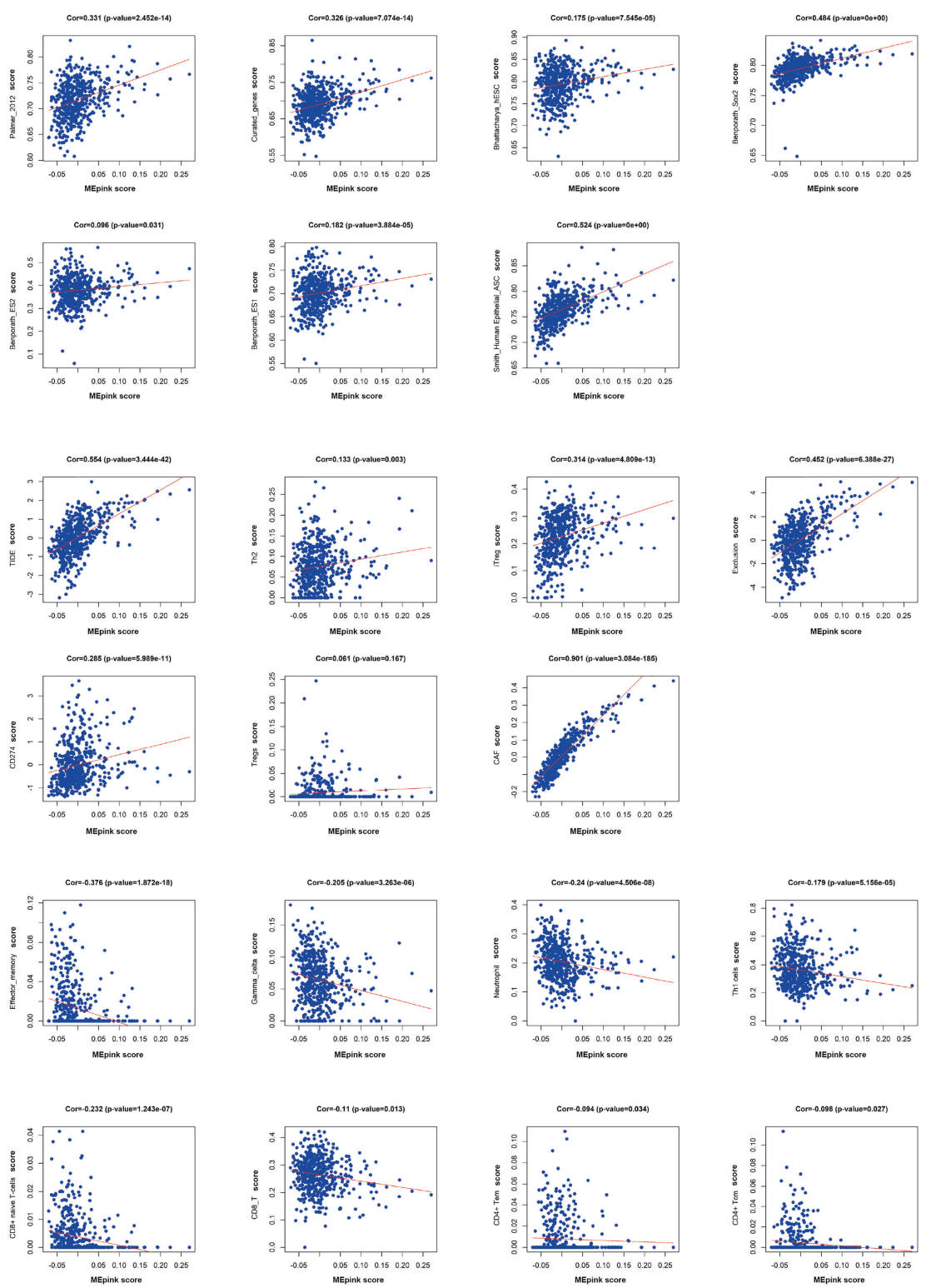

FIGURE 4 | Correlation of significant genes in pink modules with stemness and immunotherapy resistance. (A) Correlation analysis of pink modules with markers of cancer stemness. (B) Correlation analysis of pink modules with immune suppressive markers. (C) Correlation analysis of pink modules with immune stimulative markers. Cor, correlation.

ECM can damage the activation and proliferation of T cells, thus facilitating CSCs survival and inducing immunotherapy resistance (51). Peng et al. demonstrated that LAIR1-SHP-1 pathway regulated the increased collagen levels and exhausted $\mathrm{CD}^{+} \mathrm{T}$ cell subpopulations in lung tumor tissues, and mediated the resistance to PD-1/PD-L1 blockade (50). EMT increases tumor initiation and potential for metastasis, as well as resistance to several treatments (52). EMT-associated gene signatures were collectively described as features of intrinsic anti-PD-1 resistance (53). Some studies linked PD-L1 upregulation with EMT, which is also a feature closely related to CSCs $(54,55)$. Positive correlations were found between
EMT-related genes and inhibitory immune molecules (e.g., PD-L1/ 2, PD-1, CTLA-4) and Tregs in LUAD patients $(56,57)$. A transcriptomic meta-analysis of breast cancer patients showed that PD-L1 regulated the expression of OCT4A, Nanog, and BMI1 through AKT signaling (58). Thus, EMT is considered to be a potential mechanism in immune escape.

Pathway enrichment analysis in signaling pathways pointed out that genes in the pink module were en1riched in MAPK, JAK-STAT, PI3K, RAS, Notch, Wnt signaling pathways (Figure 3E). These pathways have been reported to be activated in immunotherapy resistance. For example, 
A

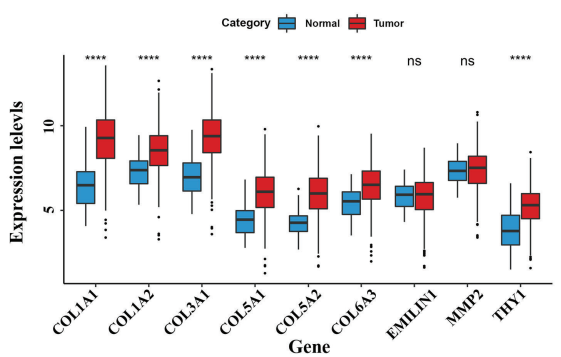

B
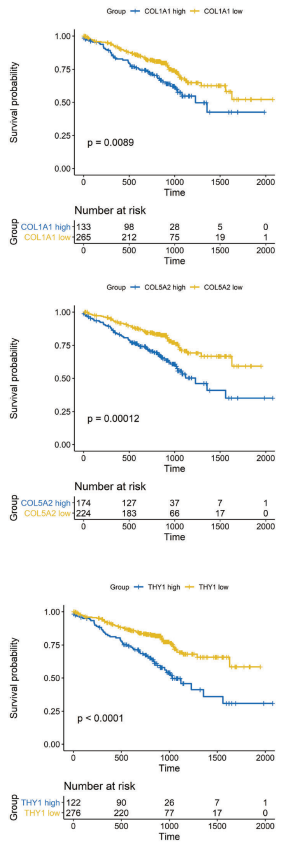
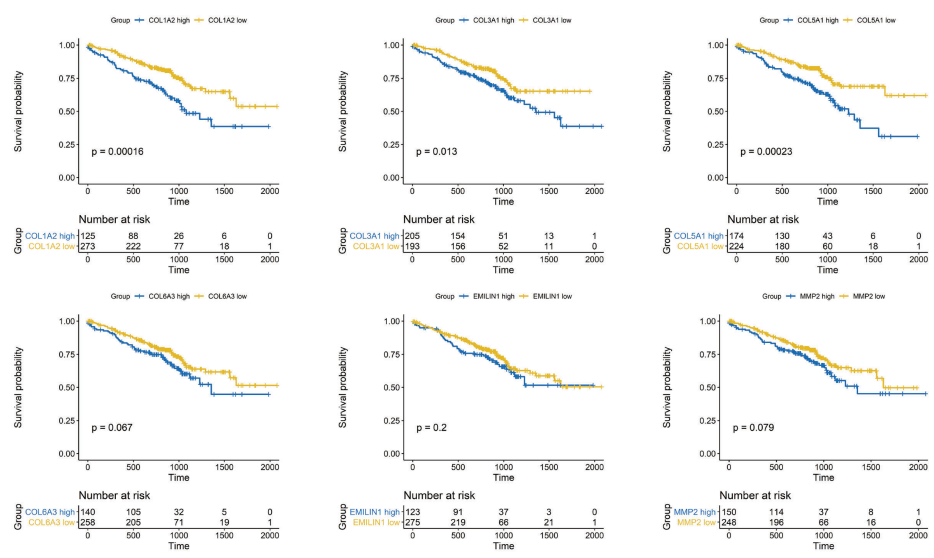

FIGURE 5 | Clinical characteristics of hub genes in pink module. (A) mRNA expressions of nine hub genes in cancer tissues of LUAD and normal lung tissues. (B) Survival plots of the significant genes by Kaplan Meier test. The data were obtained from the GEO website. ns, no significance; ${ }^{\star \star \star \star} p<0.001$.

dysregulation of MAPK pathways has been linked with immunesilencing phenotypes in breast cancer and is associated with treatment resistance (59). Activation of the JAK-STAT signaling pathway inhibits cytotoxic $\mathrm{T}$ lymphocytes and counteracts the antitumor effects of anti-PD-1 immunotherapy in pancreatic cancer (60). RAS-related oncogenesis could increase the level of PD-L1, eliminate antigen presentation, and alter the expressions of cytokines, thus leading to immune evasion and immunotherapy resistance (61). The research implies a close relationship between immunotherapy resistance and cancer stemness. Further analysis on the immune infiltration patterns of the pink module also confirms these hypotheses. We found that genes in the pink module were positively related with immune suppressive cells (Tregs, Tr1, Tr2, CAF), molecules (CD274), and two critical characteristics in immune evasion (Exclusion and Dysfunction) (Figure 4B). Meanwhile, the immune-activated cells and markers, such as antitumor $\mathrm{T}$ cells, neutrophils, Th1 cells, and gamma-delta $\mathrm{T}$ cells, were downregulated in the pink module (Figure 4C). These results support the hypothesis that the genes in the pink module can be used as potential markers or targets in identifying and eliminating malignant tumors.

To ascertain specific genes involved in ICI resistance, we selected nine genes (COL1A1, COL1A2, COL3A1, COL5A1, COL5A2, COL6A3, EMILIN1, MMP2, THY1) for further analysis. These genes are stemness-related and were predicted to be associated with the clinical outcomes of LUAD patients receiving immunotherapy. Expression analysis showed that these genes were more highly expressed in LUAD tumor tissues compared with normal tissues (Figure 5A) except for EMILIN1 and MMP2. Moreover, COL1A1, COL1A2, COL3A1, COL5A1, COL5A2, THY1 exhibited a more promising outcome in more lowly expressed group (Figure 5B). Among them, THY1 ranked first based on GS.TIDE. THY1 (CD90) is a glycosylphosphatidylinositol-anchored glycoprotein and is mainly expressed on blood stem cells, activated microvascular endothelial cells (ECs), and fibroblasts (62). It could be used as a biomarker in stem cell isolation. THY1 is an important protein in 
A

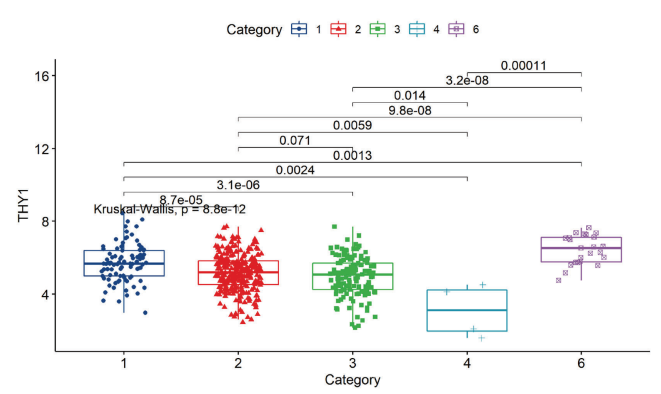

C
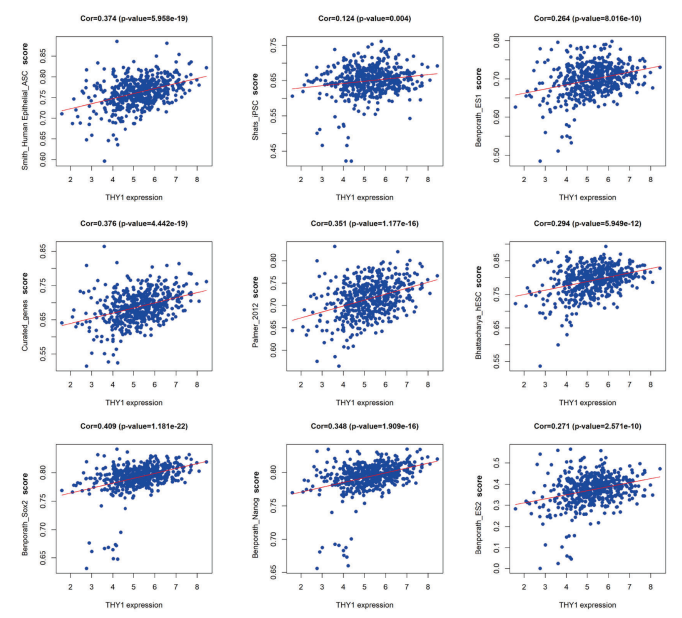

B

D
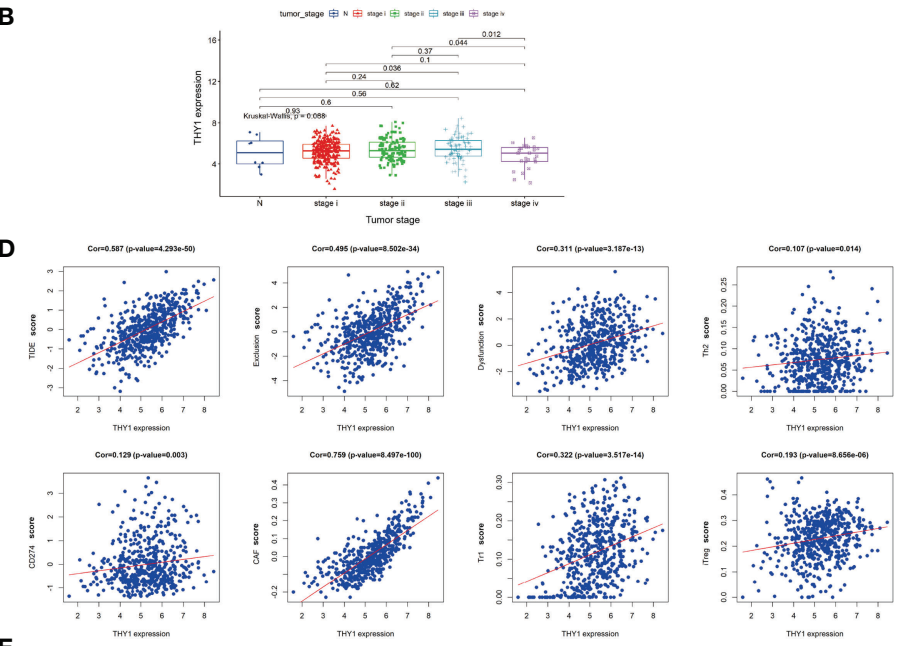

E
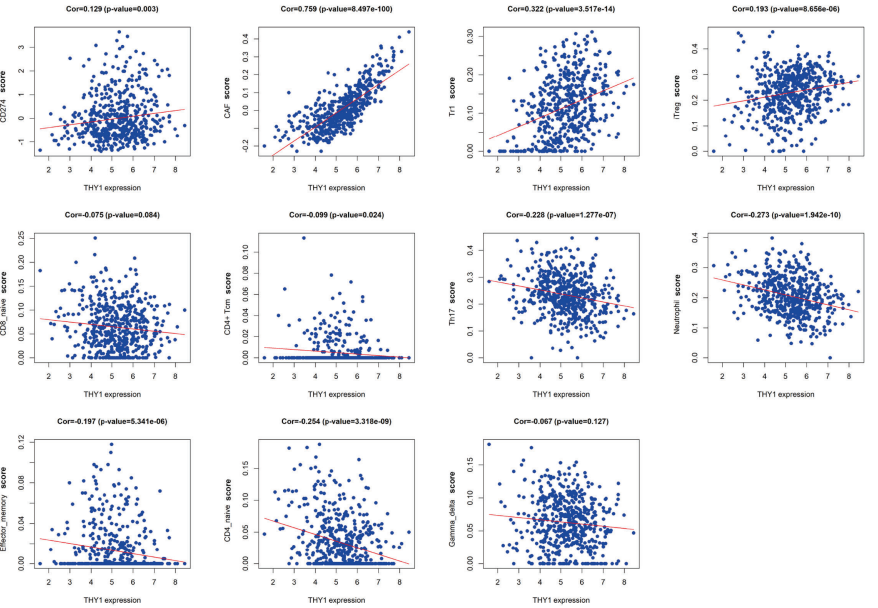

FIGURE 6 | Characteristics of THY1 in LUAD patients. (A) THY1 expression of LUAD patients in six immune subtypes: C1, wound healing; C2, IFN- $\gamma$ dominant; C3, inflammatory; C4, lymphocyte depleted; and C6. TGF-b dominant. (B) mRNA expression levels of THY1 in different tumor clinical stages (N and stage i-iv) of LUAD patients. (C) Correlation analysis of THY1 and various cancer stemness features. (D) Correlation analysis of THY1 with immunosuppressive markers. (E) Correlation analysis of THY1 with immunoactivating markers. Cor, correlation.

malignant tumors and is considered a candidate marker of CSCs with highly tumorigenic and metastatic potential due to its regulation of cell-cell interactions, cell-matrix interactions, cellular adhesion, and migration $(63,64)$. Transcriptional analysis of mRNA profiles in $\mathrm{CD}^{+} 0^{+}$esophageal CSCs showed that CD90-mediated metastasis occurs at least partially via the dysregulation of EMT and matrix metalloproteins (64). Strong correlations between THY1 and stemness markers were also found in LUAD according to our research (Figure 6C). Except for tumor cells, THY1 has also been observed in stromal cells, like ECs and CAFs, but not $\mathrm{CD} 45^{+}$cells $(65,66)$. Co-localization of THY1 and CD31 implies a possibility that CSCs may reside in the endothelial niche, facilitate vascular formation, and receive supportive signals via direct contacts with ECs (67-69). THY1 can influence tumor-infiltrating immune cells. For example, physical interactions between tumor-associated macrophages and CSCs are associated with EMT and could be regulated by CD90 and EphA4 (48). Similarly, THY1 was observed to be positively correlated with immunosuppressive markers, especially TIDE score and T cell exclusion and dysfunction (Figure 6D). However, research on THY1 in LUAD is limited. High expressions of THY1 and CD44 influence the relapse-free survival in LUAD patients (70). In our analysis, THY1 was highly expressed in the wound-healing subtype and TGF- $\beta$ dominant subtype in LUAD patient (Figure 6A). These two subtypes are related to the immunosuppressive phenotype and poor prognosis. Furthermore, THY1 was associated with tumor metastasis, tumor category, and tumor stage in LUAD, which implies that THY1 has an immunosuppressive role in TME and might be responsible for immunotherapy resistance (Figure 6C). To verify this hypothesis, we detected the expression of THY1 in LUAD patients who received PD-1 $\mathrm{mAb}$. THY1 was highly correlated with the effectiveness of PD$1 \mathrm{mAb}$ (Figure 7). Patients in PD group had the highest expression of THY1 and SOX9, a marker of cancer stemness. These results are consistent with above analysis and strongly support the hypothesis that THY1 might be a predictive marker of immunotherapy resistance and inhibiting it may improve the effects of ICI.

However, there are still some limitations in the current research. Analysis based on a public dataset cannot confirm this result, and the number of LUAD patients receiving PD-1 $\mathrm{mAb}$ included in the analysis is small. Moreover, underlying mechanisms of this phenomenon need further research. Therefore, large sample size verification and well-designed biological studies are needed to further confirm our findings. 
A

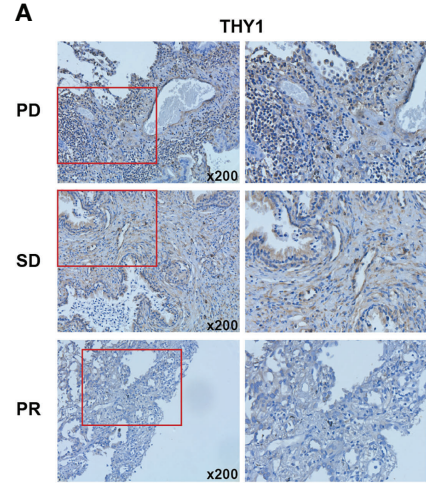

C

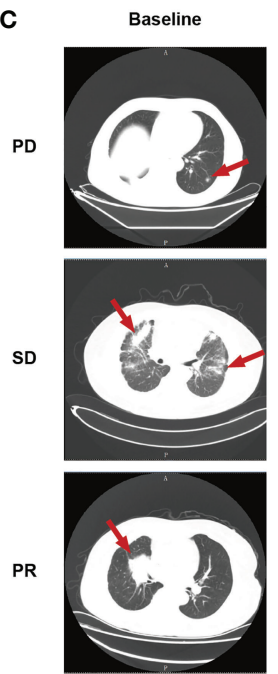

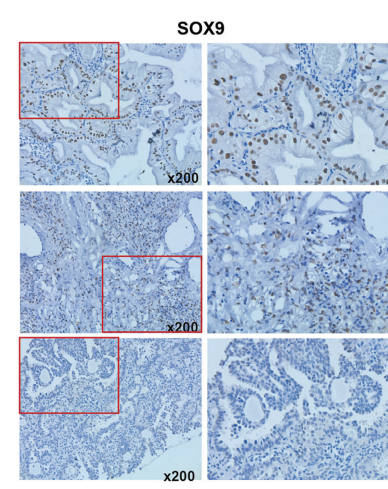
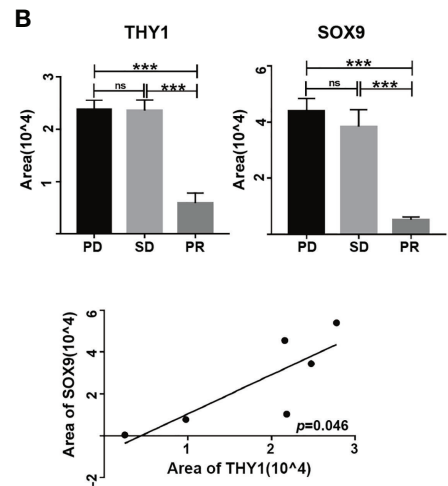

Second

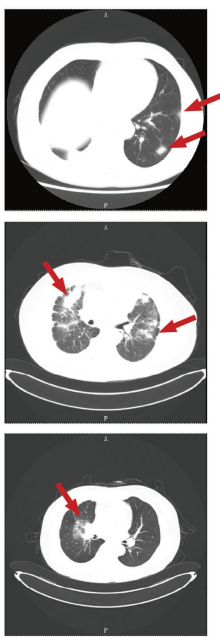

FIGURE 7 | The expression levels of THY1 are significantly associated with SOX9 and efficiency of PD-1 mAb treated LUAD patients. (A) Representative immunohistochemical staining of THY1 and SOX9 on tumor tissues of LUAD patient receiving PD-1 mAb treatment. Original magnification, 200x. (B) Statistic analysis of immunohistochemical staining score of THY1 and SOX9. Correlation analysis of the immunohistochemical staining score of THY1 and SOX9. ns, no significance; ${ }^{\star \star \star} p<$ 0.001. (C) Representative CT results of LUAD patients before and after receiving PD-1 mAb treatment. PD, progressive disease; SD, stable disease; PR, partial response.

\section{CONCLUSION}

In summary, we identified nine hub genes (COL1A1, COL1A2, COL3A1, COL5A1, COL5A2, COL6A3, EMILIN1, MMP2, THY1) as potential participants in implying cancer stemness and immunotherapy resistance. Among them, THY1, which was verified in clinical samples, was associated with the resistance of ICI treatment and co-expressed with familiar CSCs markers. As a result, THY1 may be used as a prognostic LUAD marker; and therapy targeting THY1 may be a promising avenue in eliminating LUAD and enhancing immunotherapy.

\section{DATA AVAILABILITY STATEMENT}

Publicly available datasets were analyzed in this study. This data can be found here: https://www.cancer.gov/tcga and https:// www.ncbi.nlm.nih.gov/geo/.

\section{ETHICS STATEMENT}

The studies involving human participants were reviewed and approved by the Ethics Committee of the First Affiliated Hospital of Zhengzhou University. The patients/participants provided their written informed consent to participate in this study. Written informed consent was obtained from the individual(s) for the publication of any potentially identifiable images or data included in this article.

\section{AUTHOR CONTRIBUTIONS}

YZ and LY designed the study and drafted the manuscript. WY and FL prepared the tables and figures and drafted the manuscript. All authors participated in revising the manuscript. All authors contributed to the article and approved the submitted version. 


\section{FUNDING}

This work was supported by grants from the National Natural Science Foundation of China (Nos. 91942314, U1804281, 82072578), the State's
Key Project of Research and Development Plan (No. 2021YFE0110600), the major public welfare projects in Henan Province (201300310400), and the outstanding young talents in science and technology innovation in Henan Province (YXK2020027).

\section{REFERENCES}

1. Dunn GP, Bruce AT, Ikeda H, Old LJ, Schreiber RD. Cancer Immunoediting: From Immunosurveillance to Tumor Escape. Nat Immunol (2002) 3:991-8. doi: 10.1038/ni1102-991

2. van der Woude LL, Gorris MAJ, Halilovic A, Figdor CG, de Vries IJM. Migrating Into the Tumor: A Roadmap for T Cells. Trends Cancer (2017) 3:797-808. doi: 10.1016/j.trecan.2017.09.006

3. Marin-Acevedo JA, Kimbrough EO, Lou Y. Next Generation of Immune Checkpoint Inhibitors and Beyond. J Hematol Oncol (2021) 14:45. doi: 10.1186/s13045-021-01056-8

4. Ribas A, Wolchok JD. Cancer Immunotherapy Using Checkpoint Blockade. Science (2018) 359:1350-55. doi: 10.1126/science.aar4060

5. Larkin J, Chiarion-Sileni V, Gonzalez R, Grob JJ, Cowey CL, Lao CD, et al. Combined Nivolumab and Ipilimumab or Monotherapy in Untreated Melanoma. N Engl J Med (2015) 373:23-34. doi: 10.1056/NEJMoa1504030

6. Larkin J, Chiarion-Sileni V, Gonzalez R, Grob JJ, Rutkowski P, Lao CD, et al. Five-Year Survival With Combined Nivolumab and Ipilimumab in Advanced Melanoma. N Engl J Med (2019) 381:1535-46. doi: 10.1056/NEJMoa1910836

7. Reck M, Rodriguez-Abreu D, Robinson AG, Hui R, Csoszi T, Fulop A, et al. Pembrolizumab Versus Chemotherapy for PD-L1-Positive Non-Small-Cell Lung Cancer. N Engl J Med (2016) 375:1823-33. doi: 10.1056/NEJMoa 1606774

8. Mok TSK, Wu YL, Kudaba I, Kowalski DM, Cho BC, Turna HZ, et al. Pembrolizumab Versus Chemotherapy for Previously Untreated, PD-L1Expressing, Locally Advanced or Metastatic non-Small-Cell Lung Cancer (KEYNOTE-042): A Randomised, Open-Label, Controlled, Phase 3 Trial. Lancet (2019) 393:1819-30. doi: 10.1016/S0140-6736(18)32409-7

9. Hellmann MD, Paz-Ares L, Bernabe Caro R, Zurawski B, Kim SW, Carcereny Costa E, et al. Nivolumab Plus Ipilimumab in Advanced Non-Small-Cell Lung Cancer. N Engl J Med (2019) 381(21):2020-31. doi: 10.1056/NEJMoa1910231

10. Anichini A, Perotti VE, Sgambelluri F, Mortarini R. Immune Escape Mechanisms in Non Small Cell Lung Cancer. Cancers (Basel) (2020) 12 (12):3605. doi: 10.3390/cancers 12123605

11. Nowicki TS, Hu-Lieskovan S, Ribas A. Mechanisms of Resistance to PD-1 and PD-L1 Blockade. Cancer J (2018) 24:47-53. doi: 10.1097/PPO.00000000 00000303

12. Lim SY, Rizos H. Immune Cell Profiling in the Age of Immune Checkpoint Inhibitors: Implications for Biomarker Discovery and Understanding of Resistance Mechanisms. Mamm Genome (2018) 29:866-78. doi: 10.1007/ s00335-018-9757-4

13. Joyce JA, Fearon DT. T Cell Exclusion, Immune Privilege, and the Tumor Microenvironment. Science (2015) 348:74-80. doi: 10.1126/science.aaa6204

14. Spranger S, Gajewski TF. Tumor-Intrinsic Oncogene Pathways Mediating Immune Avoidance. Oncoimmunology (2016) 5:e1086862. doi: 10.1080/ 2162402X.2015.1086862

15. Jiang P, Gu S, Pan D, Fu J, Sahu A, Hu X, et al. Signatures of T Cell Dysfunction and Exclusion Predict Cancer Immunotherapy Response. Nat Med (2018) 24:1550-58. doi: 10.1038/s41591-018-0136-1

16. Presson AP, Yoon NK, Bagryanova L, Mah V, Alavi M, Maresh EL, et al. Protein Expression Based Multimarker Analysis of Breast Cancer Samples. BMC Cancer (2011) 11:230. doi: 10.1186/1471-2407-11-230

17. Liguori M, Digifico E, Vacchini A, Avigni R, Colombo FS, Borroni EM, et al. The Soluble Glycoprotein NMB (GPNMB) Produced by Macrophages Induces Cancer Stemness and Metastasis via CD44 and IL-33. Cell Mol Immunol (2021) 18:711-22. doi: 10.1038/s41423-020-0501-0

18. Wang C, Yang Y, Yin L, Wei N, Hong T, Sun Z, et al. Novel Potential Biomarkers Associated With Epithelial to Mesenchymal Transition and Bladder Cancer Prognosis Identified by Integrated Bioinformatic Analysis. Front Oncol (2020) 10:931. doi: 10.3389/fonc.2020.00931
19. Weygant N, Ge Y, Westphalen CB, Ma WW, Vega KJ. Role of the Microenvironment in Gastrointestinal Tumors. J Oncol (2019) 2019:2153413. doi: $10.1155 / 2019 / 2153413$

20. Wei J, Barr J, Kong LY, Wang Y, Wu A, Sharma AK, et al. Glioblastoma Cancer-Initiating Cells Inhibit T-Cell Proliferation and Effector Responses by the Signal Transducers and Activators of Transcription 3 Pathway. Mol Cancer Ther (2010) 9:67-78. doi: 10.1158/1535-7163.MCT-09-0734

21. Smith BA, Balanis NG, Nanjundiah A, Sheu KM, Tsai BL, Zhang Q, et al. A Human Adult Stem Cell Signature Marks Aggressive Variants Across Epithelial Cancers. Cell Rep (2018) 24:3353-66.e5. doi: 10.1016/ j.celrep.2018.08.062

22. Malta TM, Sokolov A, Gentles AJ, Burzykowski T, Poisson L, Weinstein JN, et al. Machine Learning Identifies Stemness Features Associated With Oncogenic Dedifferentiation. Cell (2018) 173:338-54.e15. doi: 10.1016/ j.cell.2018.03.034

23. Palmer NP, Schmid PR, Berger B, Kohane IS. A Gene Expression Profile of Stem Cell Pluripotentiality and Differentiation is Conserved Across Diverse Solid and Hematopoietic Cancers. Genome Biol (2012) 13:R71. doi: 10.1186/ gb-2012-13-8-r71

24. Miranda A, Hamilton PT, Zhang AW, Pattnaik S, Becht E, Mezheyeuski A, et al. Cancer Stemness, Intratumoral Heterogeneity, and Immune Response Across Cancers. Proc Natl Acad Sci U.S.A. (2019) 116:9020-29. doi: 10.1073/ pnas. 1818210116

25. Wu YC, Tang SJ, Sun GH, Sun KH. CXCR7 Mediates TGFbeta1-Promoted EMT and Tumor-Initiating Features in Lung Cancer. Oncogene (2016) 35:2123-32. doi: 10.1038/onc.2015.274

26. Saha S, Mukherjee S, Khan P, Kajal K, Mazumdar M, Manna A, et al. Aspirin Suppresses the Acquisition of Chemoresistance in Breast Cancer by Disrupting an NFkappaB-IL6 Signaling Axis Responsible for the Generation of Cancer Stem Cells. Cancer Res (2016) 76:2000-12. doi: 10.1158/00085472.CAN-15-1360

27. Singh SP, Singh R, Gupta OP, Gupta S, Brahma Bhatt ML. Immunotherapy: Newer Therapeutic Armamentarium Against Cancer Stem Cells. J Oncol (2020) 2020:3963561. doi: 10.1155/2020/3963561

28. Chen H, He X. The Convergent Cancer Evolution Toward a Single Cellular Destination. Mol Biol Evol (2016) 33:4-12. doi: 10.1093/molbev/msv212

29. Shats I, Gatza ML, Chang JT, Mori S, Wang J, Rich J, et al. Using a Stem CellBased Signature to Guide Therapeutic Selection in Cancer. Cancer Res (2011) 71:1772-80. doi: 10.1158/0008-5472.CAN-10-1735

30. Ben-Porath I, Thomson MW, Carey VJ, Ge R, Bell GW, Regev A, et al. An Embryonic Stem Cell-Like Gene Expression Signature in Poorly Differentiated Aggressive Human Tumors. Nat Genet (2008) 40:499-507. doi: 10.1038/ng.127

31. Bhattacharya B, Miura T, Brandenberger R, Mejido J, Luo Y, Yang AX, et al. Gene Expression in Human Embryonic Stem Cell Lines: Unique Molecular Signature. Blood (2004) 103:2956-64. doi: 10.1182/blood-2003-09-3314

32. Almeida LG, Sakabe NJ, deOliveira AR, Silva MC, Mundstein AS, Cohen T, et al. CTdatabase: A Knowledge-Base of High-Throughput and Curated Data on Cancer-Testis Antigens. Nucleic Acids Res (2009) 37:D816-9. doi: 10.1093/ nar/gkn673

33. Langfelder P, Horvath S. WGCNA: An R Package for Weighted Correlation Network Analysis. BMC Bioinf (2008) 9:559. doi: 10.1186/1471-2105-9-559

34. Yin L, Cai Z, Zhu B, Xu C. Identification of Key Pathways and Genes in the Dynamic Progression of HCC Based on WGCNA. Genes (Basel) (2018) 9 (2):92. doi: 10.3390/genes9020092

35. Thorsson V, Gibbs DL, Brown SD, Wolf D, Bortone DS, Ou Yang TH, et al. The Immune Landscape of Cancer. Immunity (2018) 48:812-30.e14. doi: 10.1016/j.immuni.2018.03.023

36. Yang L, Li A, Liu F, Zhao Q, Ji S, Zhu W, et al. Immune Profiling Reveals Molecular Classification and Characteristic in Urothelial Bladder Cancer. Front Cell Dev Biol (2021) 9:596484. doi: 10.3389/fcell.2021.596484 
37. Saleh R, Elkord E. Acquired Resistance to Cancer Immunotherapy: Role of Tumor-Mediated Immunosuppression. Semin Cancer Biol (2020) 65:13-27. doi: 10.1016/j.semcancer.2019.07.017

38. Christin JR, Wang C, Chung CY, Liu Y, Dravis C, Tang W, et al. Stem Cell Determinant SOX9 Promotes Lineage Plasticity and Progression in Basal-Like Breast Cancer. Cell Rep (2020) 31:107742. doi: 10.1016/j.celrep.2020.107742

39. Killock D. Signatures IMPRES and Might Turn the TIDE in Predicting Responses. Nat Rev Clin Oncol (2018) 15:654. doi: 10.1038/s41571-0180088-x

40. Bao S, Wu Q, McLendon RE, Hao Y, Shi Q, Hjelmeland AB, et al. Glioma Stem Cells Promote Radioresistance by Preferential Activation of the DNA Damage Response. Nature (2006) 444:756-60. doi: 10.1038/nature05236

41. Diehn M, Clarke MF. Cancer Stem Cells and Radiotherapy: New Insights Into Tumor Radioresistance. J Natl Cancer Inst (2006) 98:1755-7. doi: 10.1093/ jnci/djj505

42. Irvin DK, Jouanneau E, Duvall G, Zhang XX, Zhai Y, Sarayba D, et al. T Cells Enhance Stem-Like Properties and Conditional Malignancy in Gliomas. PloS One (2010) 5:e10974. doi: 10.1371/journal.pone.0010974

43. Maccalli C, Parmiani G, Ferrone S. Immunomodulating and Immunoresistance Properties of Cancer-Initiating Cells: Implications for the Clinical Success of Immunotherapy. Immunol Invest (2017) 46:221-38. doi: 10.1080/08820139.2017.1280051

44. Mani SA, Guo W, Liao MJ, Eaton EN, Ayyanan A, Zhou AY, et al. The Epithelial-Mesenchymal Transition Generates Cells With Properties of Stem Cells. Cell (2008) 133:704-15. doi: 10.1016/j.cell.2008.03.027

45. Pankova D, Jiang Y, Chatzifrangkeskou M, Vendrell I, Buzzelli J, Ryan A, et al. RASSF1A Controls Tissue Stiffness and Cancer Stem-Like Cells in Lung Adenocarcinoma. EMBO J (2019) 38:e100532. doi: 10.15252/embj. 2018100532

46. Wang L, Zuo X, Xie K, Wei D. The Role of CD44 and Cancer Stem Cells. Methods Mol Biol (2018) 1692:31-42. doi: 10.1007/978-1-4939-7401-6_3

47. Dituri F, Mazzocca A, Giannelli G, Antonaci S. PI3K Functions in Cancer Progression, Anticancer Immunity and Immune Evasion by Tumors. Clin Dev Immunol (2011) 2011:947858. doi: 10.1155/2011/947858

48. Lu H, Clauser KR, Tam WL, Frose J, Ye X, Eaton EN, et al. A Breast Cancer Stem Cell Niche Supported by Juxtacrine Signalling From Monocytes and Macrophages. Nat Cell Biol (2014) 16:1105-17. doi: 10.1038/ncb3041

49. Bollyky PL, Wu RP, Falk BA, Lord JD, Long SA, Preisinger A, et al. ECM Components Guide IL-10 Producing Regulatory T-Cell (TR1) Induction From Effector Memory T-Cell Precursors. Proc Natl Acad Sci USA (2011) 108:7938-43. doi: 10.1073/pnas.1017360108

50. Peng DH, Rodriguez BL, Diao L, Chen L, Wang J, Byers LA, et al. Collagen Promotes Anti-PD-1/PD-L1 Resistance in Cancer Through LAIR1Dependent CD8(+) T Cell Exhaustion. Nat Commun (2020) 11:4520. doi: 10.1038/s41467-020-18298-8

51. Di Tomaso T, Mazzoleni S, Wang E, Sovena G, Clavenna D, Franzin A, et al. Immunobiological Characterization of Cancer Stem Cells Isolated From Glioblastoma Patients. Clin Cancer Res (2010) 16:800-13. doi: 10.1158/ 1078-0432.CCR-09-2730

52. Dongre A, Weinberg RA. New Insights Into the Mechanisms of EpithelialMesenchymal Transition and Implications for Cancer. Nat Rev Mol Cell Biol (2019) 20:69-84. doi: 10.1038/s41580-018-0080-4

53. Hugo W, Zaretsky JM, Sun L, Song C, Moreno BH, Hu-Lieskovan S, et al. Genomic and Transcriptomic Features of Response to Anti-PD-1 Therapy in Metastatic Melanoma. Cell (2016) 165:35-44. doi: 10.1016/j.cell.2016.02.065

54. Zhi Y, Mou Z, Chen J, He Y, Dong H, Fu X, et al. B7H1 Expression and EpithelialTo-Mesenchymal Transition Phenotypes on Colorectal Cancer Stem-Like Cells. PloS One (2015) 10:e0135528. doi: 10.1371/journal.pone.0135528

55. Lee Y, Shin JH, Longmire M, Wang H, Kohrt HE, Chang HY, et al. CD44+ Cells in Head and Neck Squamous Cell Carcinoma Suppress T-Cell-Mediated Immunity by Selective Constitutive and Inducible Expression of PD-L1. Clin Cancer Res (2016) 22:3571-81. doi: 10.1158/1078-0432.CCR-15-2665

56. Chen L, Gibbons DL, Goswami S, Cortez MA, Ahn YH, Byers LA, et al. Metastasis is Regulated via microRNA-200/ZEB1 Axis Control of Tumour Cell PD-L1 Expression and Intratumoral Immunosuppression. Nat Commun (2014) 5:5241. doi: 10.1038/ncomms6241

57. Lou Y, Diao L, Cuentas ER, Denning WL, Chen L, Fan YH, et al. EpithelialMesenchymal Transition Is Associated With a Distinct Tumor
Microenvironment Including Elevation of Inflammatory Signals and Multiple Immune Checkpoints in Lung Adenocarcinoma. Clin Cancer Res (2016) 22:3630-42. doi: 10.1158/1078-0432.CCR-15-1434

58. Almozyan S, Colak D, Mansour F, Alaiya A, Al-Harazi O, Qattan A, et al. PDL1 Promotes OCT4 and Nanog Expression in Breast Cancer Stem Cells by Sustaining PI3K/AKT Pathway Activation. Int J Cancer (2017) 141:1402-12. doi: $10.1002 /$ ijc.30834

59. Bedognetti D, Roelands J, Decock J, Wang E, Hendrickx W. The MAPK Hypothesis: Immune-Regulatory Effects of MAPK-Pathway Genetic Dysregulations and Implications for Breast Cancer Immunotherapy. Emerg Top Life Sci (2017) 1:429-45. doi: 10.1042/ETLS20170142

60. Lu C, Talukder A, Savage NM, Singh N, Liu K. JAK-STAT-Mediated Chronic Inflammation Impairs Cytotoxic T Lymphocyte Activation to Decrease AntiPD-1 Immunotherapy Efficacy in Pancreatic Cancer. Oncoimmunology (2017) 6:e1291106. doi: 10.1080/2162402X.2017.1291106

61. Ward AB, Keeton AB, Chen X, Mattox TE, Coley AB, Maxuitenko YY, et al. Enhancing Anticancer Activity of Checkpoint Immunotherapy by Targeting RAS. MedComm (Beijing) (2020) 1:121-28. doi: 10.1002/mco2.10

62. Dennis JE, Esterly K, Awadallah A, Parrish CR, Poynter GM, Goltry KL. Clinical-Scale Expansion of a Mixed Population of Bone-Marrow-Derived Stem and Progenitor Cells for Potential Use in Bone-Tissue Regeneration. Stem Cells (2007) 25:2575-82. doi: 10.1634/stemcells.2007-0204

63. Yang ZF, Ho DW, Ng MN, Lau CK, Yu WC, Ngai P, et al. Significance of CD90+ Cancer Stem Cells in Human Liver Cancer. Cancer Cell (2008) 13:153-66. doi: 10.1016/j.ccr.2008.01.013

64. Tang KH, Dai YD, Tong M, Chan YP, Kwan PS, Fu L, et al. A CD90(+) Tumor-Initiating Cell Population With an Aggressive Signature and Metastatic Capacity in Esophageal Cancer. Cancer Res (2013) 73:2322-32. doi: 10.1158/0008-5472.CAN-12-2991

65. Sukowati CH, Anfuso B, Torre G, Francalanci P, Croce LS, Tiribelli C. The Expression of CD90/Thy-1 in Hepatocellular Carcinoma: An In Vivo and In Vitro Study. PloS One (2013) 8:e76830. doi: 10.1371/journal.pone.0076830

66. Samaniego R, Estecha A, Relloso M, Longo N, Escat JL, Longo-Imedio I, et al. Mesenchymal Contribution to Recruitment, Infiltration, and Positioning of Leukocytes in Human Melanoma Tissues. J Invest Dermatol (2013) 133:225564. doi: $10.1038 /$ jid.2013.88

67. He J, Liu Y, Zhu T, Zhu J, Dimeco F, Vescovi AL, et al. CD90 is Identified as a Candidate Marker for Cancer Stem Cells in Primary High-Grade Gliomas Using Tissue Microarrays. Mol Cell Proteomics (2012) 11:M111 010744. doi: 10.1074/mcp.M111.010744

68. Zhu J, Thakolwiboon S, Liu X, Zhang M, Lubman DM. Overexpression of CD90 (Thy-1) in Pancreatic Adenocarcinoma Present in the Tumor Microenvironment. PloS One (2014) 9:e115507. doi: 10.1371/journal. pone. 0115507

69. Lasorella A, Benezra R, Iavarone A. The ID Proteins: Master Regulators of Cancer Stem Cells and Tumour Aggressiveness. Nat Rev Cancer (2014) 14:7791. doi: $10.1038 / \mathrm{nrc} 3638$

70. Mehta K, Moravcikova E, McFall D, Luketich JD, Pennathur A, Donnenberg $\mathrm{AD}$, et al. The Mesenchymal State Predicts Poor Disease-Free Survival in Resectable Non-Small Cell Lung Cancer. Ann Thorac Surg (2017) 104:321-28. doi: 10.1016/j.athoracsur.2017.01.091

Conflict of Interest: The authors declare that the research was conducted in the absence of any commercial or financial relationships that could be construed as a potential conflict of interest.

Publisher's Note: All claims expressed in this article are solely those of the authors and do not necessarily represent those of their affiliated organizations, or those of the publisher, the editors and the reviewers. Any product that may be evaluated in this article, or claim that may be made by its manufacturer, is not guaranteed or endorsed by the publisher.

Copyright $\odot 2022 \mathrm{Yu}, \mathrm{Liu}, \mathrm{Lei}, \mathrm{Wu}$, Yang and Zhang. This is an open-access article distributed under the terms of the Creative Commons Attribution License (CC BY). The use, distribution or reproduction in other forums is permitted, provided the original author(s) and the copyright owner(s) are credited and that the original publication in this journal is cited, in accordance with accepted academic practice. No use, distribution or reproduction is permitted which does not comply with these terms. 